\title{
Energy Consumption Comparison Between Macro-Micro and Public Femto Deployment in a Plausible LTE Network
}

\author{
Kateřina Dufková \\ Czech Technical University in \\ Prague \\ Prague, Czech Republic \\ katerina.dufkova@rdc.cz \\ Jean-Yves Le Boudec \\ EPFL, IC-LCA2 \\ $\mathrm{CH}-1015$ Lausanne, \\ Switzerland \\ jean- \\ yves.leboudec@epfl.ch
}

\author{
Miroslav Popović \\ EPFL, IC-LCA2 \\ $\mathrm{CH}-1015$ Lausanne, \\ Switzerland \\ miroslav.popovic@epfl.ch \\ Milan Bjelica \\ Faculty of Electrical \\ Engineering (ETF) \\ University of Belgrade \\ Serbia \\ milan@etf.rs
}

\author{
Ramin Khalili \\ EPFL, IC-LCA2 \\ $\mathrm{CH}-1015$ Lausanne, \\ Switzerland \\ ramin.khalili@epfl.ch \\ Lukáš Kencl \\ Czech Technical University in \\ Prague \\ Prague, Czech Republic \\ lukas.kencl@rdc.cz
}

\begin{abstract}
We study the energy consumptions of two strategies that increase the capacity of an LTE network: (1) the deployment of redundant macro and micro base stations by the operator at locations where the traffic is high, and (2) the deployment of publicly accessible femto base stations by home users. Previous studies show the deployment of publicly accessible residential femto base stations is considerably more energy efficient; however, the results are proposed using an abstracted model of LTE networks, where the coverage constraint was neglected in the study, as well as some other important physical and traffic layer specifications of LTE networks. We study a realistic scenario where coverage is provided by a set of non-redundant macro-micro base stations and additional capacity is provided by redundant macro-micro base stations or by femto base stations. We quantify the energy consumption of macro-micro and femto deployment strategies by using a simulation of a plausible LTE deployment in a mid-size metropolitan area, based on data obtained from an operator and using detailed models of heterogeneous devices, traffic, and physical layers. The metrics of interest are operator-energy-consumption/totalenergy-consumption per unit of network capacity.

For the scenarios we studied, we observe the following: (1) There is no significant difference between operator energy consumption of femto and macro-micro deployment strategies. From the point of view of society, i.e. total energy
\end{abstract}

\footnotetext{
* This research has received funding from the EU 7th Framework Programme (FP7/2007-2013) under grant agreement n. 257740 (Network of Excellence "TREND") and from the Serbian Ministry of Science under project n. TR32028.
}

consumption, macro-micro deployment is even more energy efficient in some cases. This differs from the previous findings, which compared the energy consumption of femto and macro-micro deployment strategies, and found that femto deployment is considerably more energy efficient. (2) The deployment of femto base stations has a positive effect on mobile-terminal energy consumption; however, it is not significant compared to the macro-micro deployment strategy. (3) The energy saving that could be obtained by making macro and micro base stations more energy proportional is much higher than that of femto deployment.

\section{Categories and Subject Descriptors}

C.2.1 [Network Architecture and Design]: Wireless communications; C.4 [Performance of Systems]: Modeling Techniques

\section{General Terms}

Performance

\section{Keywords}

LTE networks, energy, performance evaluation

\section{INTRODUCTION}

Great attention is currently devoted to the energy efficiency of future wireless networks [1], for multiple reasons: (1) the need to cut operating expenses and energy consumption of mobile telecommunication operators, where radio network represents about $80 \%$ of energy costs; (2) governments' pressure to reduce the industrial carbon footprint; (3) the need to save on primary energy consumption due to growing global demand; and (4) exponential growth of mobile data traffic [2], which requires higher wireless network capacity and consequently an increase in consumed energy.

One of the recent developments in mobile networks is the introduction of femto base stations [3]. Femto base stations are low-power, low-cost, user-deployed devices, designed for use in residential or enterprise environments. Although femto base stations are expected to initially be deployed with access restricted to private users only, Claussen 
et al. show in [4] that there is a huge potential to reduce the energy consumption of LTE networks by using the publicly accessible residential femto base stations (which they name as pico base stations) to supplement the capacity of the macro network. In particular, they show that up to $70 \%$ energy saving can be achieved by a joint deployment of macro and femto base stations in the network compared to the case when only macro base stations are deployed.

As [4] is generally considered as a reference paper on femto base-station deployment, we devoted most of our attention to understanding its findings and their implications. We concluded that the results were obtained using an abstracted model of LTE network which assumes hypothetical network topologies where no coverage constraints are considered. Moreover, many other important specifications of LTE network's physical and traffic layer had been ignored in the study. It considers that there is a linear relationship between the number of sessions served by femto base stations and the number of femto base stations deployed, which is in contradictory with results in [5]. Furthermore, the only QoS constraint applied is the average number of users that can be served by a base station, which does not guarantee that users will receive bounded service times.

We study the energy savings due to the deployment of femto base stations by using a simulation (based on data obtained from an operator) of a plausible LTE deployment in a mid-size metropolitan area, and by using detailed models of heterogeneous devices, traffic, and physical layers. In our setting, the coverage is provided by a set of non-reduntant macro-micro base stations and additional capacity is provided by redundant macro-micro base stations or by femto base stations. Note that mobile telecommunication operators cannot rely on coverage provided by femto base stations as they do not have full control on their functionality. We compare the energy consumption of two strategies that increase the capacity of LTE macro networks:

- Macro-micro deployment is the deployment of redundant macro and micro base stations (refer to Section 2.3 for a detailed definition of redundant base stations) by operators at locations where the traffic load is heavy. Specifically, we consider the deployment of 10 macro and micro base stations whose positions are determined from the operator data.

- Femto deployment: is the deployment of publicly accessible femto base stations by home users to supplement the capacity of the macro network as proposed in [4]. We consider different number of deployed femto base stations.

We simulate a network that uses a heterogeneous set of LTE base stations, with different energy-performance trade-offs (refer to Section 2.3). The network model is constrained to always provide full coverage (refer to Section 2.3.1). The user association policy is defined such that a user will not experience a large service-delay (Section 2.4). The traffic scenario is dynamic and heterogeneous; it combines web and video traffic, which are expected to be dominating in the future (refer to Section 2.2). Users are not mobile (the effect of handovers is not considered), but dynamically appear and disappear throughout the simulation. The physical layer of LTE networks are modeled in detail as explained in Section 2.1. For modeling device power-consumption, we use empirical models observed in the literature $[4,6-8]$, where we capture the dependency of power consumption on an immediate traffic load (Section 3).

We quantify energy consumptions and capacity enhancements of two deployment strategies through simulation. The metrics of interest are operator-energy-consumption/totalenergy-consumption per unit of network capacity. For the studied scenarios, we observe the following:

- There is no significant difference between operator energy consumption of femto and macro-micro deployment strategies. From the point of view of society, i.e. total energy consumption, macro-micro deployment is even more energy efficient in some cases. This differs from the previous findings in [4], which compared the energy consumption of femto and macro-micro deployment strategies and found that femto deployment was considerably more energy efficient.

- Femto deployment has a positive effect on mobile-phone energy consumption, in accordance with findings in [9]. However, this effect is not significant compared to the case when additional macro and micro base stations are deployed in the network.

- The energy saving that could be obtained by making macro and micro base stations more energy proportional is much higher than the energy saving of femto deployment.

Finally, we study the effect of femto base-station penetrations only on the energy consumption and capacity of the network. The results show that both operator and totalenergy-consumption per unit of capacity decrease as the number of deployed femto base stations increases. We consider deployment of up to 1000 femto base stations. We expect that for higher penetrations the positive effects of femto base stations on the total-energy-consumption per unit of capacity would be diminished; however, we could not verify this hypothesis as our 2D interference model prevented us from adding more than 1000 femto base stations.

\section{MODEL AND ASSUMPTIONS}

Our goal in this paper is to compare the energy consumption of femto and redundant macro-micro deployment strategies. To understand the performance of each of these strategies better, based on data obtained from an operator, we simulate a plausible LTE deployment in a mid-size metropolitan area. We consider a heterogeneous network a co-channel deployment of macro, micro and femto base stations; we will disscuss deployment details. To the best of our knowledge, there are no representative operational data from the commercial LTE networks available, hence, some assumptions need to be made, as will be explained in this section. Throughout this paper all sensitive information from the operator is obfuscated for confidentiality reasons.

We focus on the downlink traffic of LTE networks, assuming the uplink traffic is of secondary importance for web browsing and video streaming users. However, studying the uplink could be an issue, depending on how the spectrum and/or time are shared between uplink and downlink traffic. It is not possible to ignore the case where the uplink would be the bottleneck; in fact, a good engineering policy would be to balance the resources between uplink and downlink. Further, operators may be interested in restricting uplink capacity to reduce the amount of peer-to-peer traffic. 


\subsection{Physical Layer Model}

Calculations of the signal level received from a base station is done as described by [10]

$P_{R X}[\mathrm{dBm}]=P_{\mathrm{TX} \text { subcarrier }}[\mathrm{dBm}]+A[\mathrm{~dB}]-P_{L}[\mathrm{~dB}]-\Psi[\mathrm{dB}]$,

where $P_{\mathrm{TX} \text { subcarrier }}$ is the effective transmit power per subcarrier (transmitting antenna gain taken in account). The effective transmit power per subcarrier is the Equivalent Isotropically Radiated Power in $\mathrm{dBi}$ (EIRP), i.e. it is the emitted transmission power of a theoretical isotropic antenna to produce the same peak power density as in the direction of the maximum antenna gain. $A$ is the antenna radiation pattern, $P_{L}$ is the path loss and $\Psi$ is the expected value for shadow fading.

The effective transmit-power levels per subcarrier are chosen based on the measurement results of the Swiss Federal Office of Communications [11]. According to their characterization, the radiated power is divided into the following categories: very low, low, medium, and high. In the city of interest, no sites have low radiated power and for the rest (very low, medium, high) we choose $P_{\text {TXsubcarrier }}$ equal to $25 \mathrm{dBm}, 35 \mathrm{dBm}$ and $43 \mathrm{dBm}$, respectively. For femto base stations we use $15 \mathrm{dBm}$.

We applied path-loss models from [10] (1) Urban Micro, (2) Urban Macro for micro and macro base stations in urban areas, and (3) Suburban Macro for macro base stations in suburban areas. They take into account distance, line-ofsight existence, antenna height, average building height, etc. For users indoors we assume $20 \mathrm{~dB}$ of attenuation to account for outdoor-indoor penetration loss. For femto base stations, we use the propagation model from [12].

Antenna radiation patterns are used to take into account radio signal attenuation in the direction of interest, compared to the boresight of the antenna. For omnidirectional antennas, $A=0$. All femto base stations have omnidirectional antennas, as well as some of the macro and micro base stations. For base station sectors with directed antennas, the antenna pattern is calculated by using the following equation defined in [13]

$$
A(\theta)=-\min \left[12\left(\frac{\theta}{\theta_{3 \mathrm{~dB}}}\right)^{2}, A_{m}\right],
$$

$-180^{\circ} \leq \theta \leq 180^{\circ}$ is the angle between the direction of interest and the antenna boresight, $\theta_{3 \mathrm{~dB}}=70^{\circ}$ is the $3 \mathrm{~dB}$ beamwidth and $A_{m}=30 \mathrm{~dB}$ is the maximum attenuation.

The distribution of shadow fading is log-normal and its standard deviation values are taken from [10]. We consider $5 \mathrm{MHz}$ channel bandwidth that is used by base stations.

\subsection{Traffic Model}

We extrapolate our traffic model from an operator trace file with traffic data in the observed network segment. It contains the activity type (call or SMS), time and serving sector. Every trace-file entry represents an arrival generating one session - we replicate this session-arrival sequence exactly in the simulation. We model the session duration as a random variable with a 30-minute mean. During one session, a user generates data requests randomly according to a Poisson process of intensity $\lambda$ in $\mathrm{s}^{-1}$, where the size of a data-request is random with mean of $\sigma$ bits.

We consider two types of users in the network. The first type generates web sessions and the second generates video

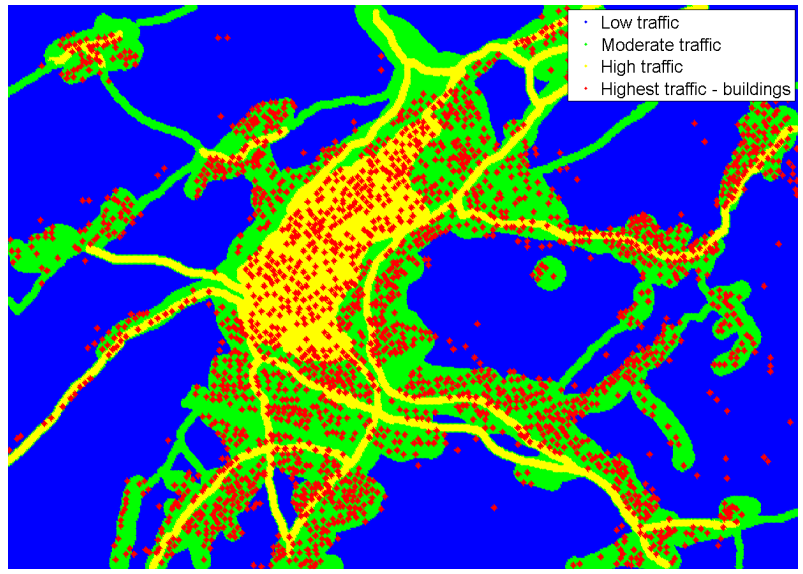

Figure 1: Region separated into areas with different traffic intensities.

traffic. Users who generated calls (resp. SMSs) in the trace generate video (resp. web) traffic in the simulator. We denote by $\left(\lambda_{w}, \sigma_{w}\right)$ and $\left(\lambda_{v}, \sigma_{v}\right)$ the mean data-request arrival rate and the mean data-request size of traffic generated by web and video users, respectively.

Let $N_{w, i}(t)$ and $N_{v, i}(t)$ be, respectively, the number of web and video users served by base-station sector $i$ at time $t$. We assume a fair sharing of the available frequency subband at base station sector $i$ among all active users [14]. We define by $R_{i}(u)$ the instantaneous feasible throughput of a user served by $i$ at relative position $u$ (we discuss in this section how to calculate $R_{i}(u)$ ). The instantaneous actual data rate of the user, therefore, is $R_{i}(u) /\left[N_{w, i}(t)+N_{v, i}(t)\right]$.

$N_{w, i}(t)$ and $N_{v, i}(t)$ behave as the number of customers in a multi-class product-form queuing network with a processor sharing service discipline [15, Ch. 8]. The class of a customer served by $i$ is defined by $u$, the costumer position relative to $i$, and its type. With the insensitivity property of multiclass processor sharing queuing models, base-station sector $i$ traffic load can be determined without knowing the fine traffic statistics $[16,17]$ :

$$
\rho_{i}=\lambda_{w} \sigma_{w} \sum_{j=1}^{N_{w, i}(t)} \frac{1}{R_{i}\left(u_{j}\right)}+\lambda_{v} \sigma_{v} \sum_{k=1}^{N_{v, i}(t)} \frac{1}{R_{i}\left(u_{k}\right)} .
$$

Base-station sector $i$ is stable if $\rho_{i} \leq 1$. As discussed in Section 2.4, we use $\rho_{i}$ to decide if a new user can be served by sector $i$, or should be blocked.

From the trace files, we know the serving sector of a user, but we do not know the exact position of the user inside the sector. To position a user within the serving sector in a realistic way, we split the whole region into four different areas based on the expected level of traffic (Figure 1). In the rural areas, the expected level of traffic is low, on the city outskirts it is medium, in the city center it is high, and the highest expected level of traffic is in the vicinity of buildings. We assign probabilities, proportional to the expected level of traffic, for the position of a user in each of these areas inside the observed sector of a base station.

To apply the $20 \mathrm{~dB}$ of outdoor-indoor penetration loss in received-signal level calculation, we need to know if a user is indoors or not. Note that from the map of the city we know the positions of the houses. We consider them as squares 


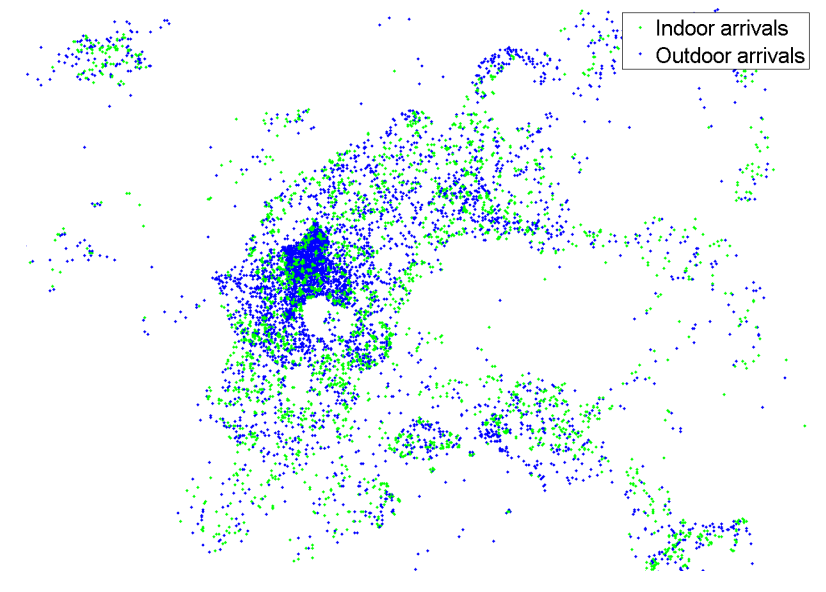

Figure 2: Position of 10000 arrivals - indoors and outdoors.

with sides of length between $20 \mathrm{~m}$ and $80 \mathrm{~m}$. If the user is inside a square, it is considered to be indoors; otherwise, he is considered to be outdoors as depicted in Figure 2.

If a base-station sector serves only one user, the feasible throughput that can be achieved by that user is given by

$$
R_{i}(u)=W \log _{2}\left(1+\gamma_{j}(u)\right),
$$

where $W=5 \mathrm{MHz}$ is the bandwidth used at $i$, and

$$
\gamma_{j}(u)=P_{i} /\left(\sum_{k \in \mathcal{I}} \rho_{k} P_{k}+N_{0}\right)
$$

is the average signal-to-interference-plus-noise ratio received by the user, $P_{i}$ being the received signal level from basestation sector $i, \mathcal{I}$ the set of interfering base-station sectors, $\rho_{k}$ the traffic intensity at interfering base station $k\left(\rho_{k} P_{k}\right.$ is the average interference per subcarrier received from interfering base station $k$ ), and $N_{0}$ the noise level over the entire bandwidth. Aforementioned signal levels are calculated as explained in Section 2.1.

\subsection{Network Model}

We abstract our network model from real network-deployment data obtained from a telecom operator. Deployment data contain positions of macro and micro base stations and antenna directions, when directed antennas are used. There are two different classes of base stations in the region:

- class A (non-redundant) base stations: These are non-redundant macro and micro base stations that provide full coverage to the users (refer to Section 2.3.1). We cannot remove or switch off a class A base station without losing coverage to some users in the region.

- class B (redundant) base stations: These redundant base stations are deployed to increase the capacity of the network. Class B base stations do not contribute to the coverage of class A base stations and are deployed by the operator at locations where traffic density is high.

We have 86 macro and micro base-station sectors on 45 sites, where 76 sectors are class A base stations (Figure 3) and 10 sectors are class B base stations, as depicted in Figure 4.

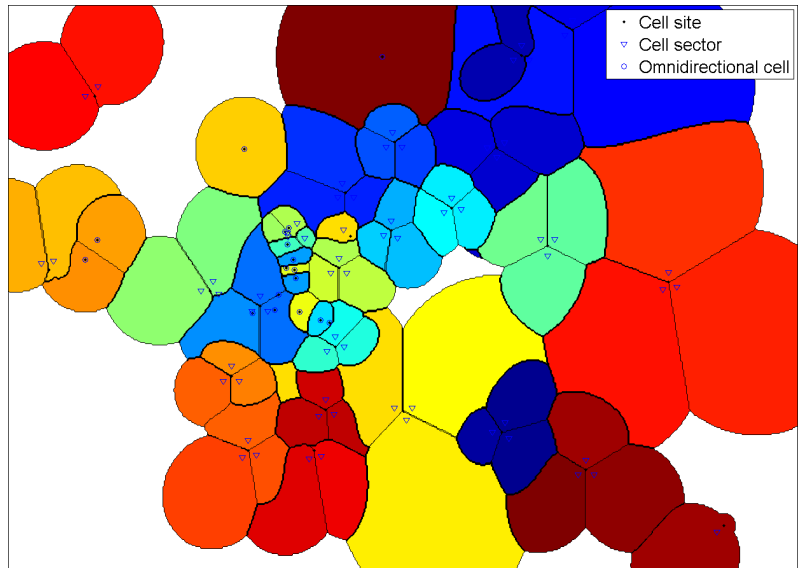

Figure 3: Coverage area of class A (non-redundant) macro and micro base-station sectors.

We consider a network of class A base stations and we quantify energy consumption and capacity enhancement of deployment of redundant class B base stations. Alternatively, we assume deployment of small femto base stations, installed by home users, together with class A base stations. Femto base stations can provide service to indoor and outdoor users. They are connected to the network operator via the home user's fixed broadband connection. We consider femto base stations as open, which means that home users allow other mobile devices to access their femto base stations. Although the "Closed User Group" model is usually assumed today for femto base stations, we believe that open femto base stations might be also an option in the future (they are called pico base stations in [4]).

Each city building is considered as a potential placeholder for a femto base station. The particular buildings where they are installed are selected uniformly. However, similarly to [4], the fraction of these buildings in certain parts of the region is proportional to the expected traffic in that part. We consider a 2D model, excluding the floor on which the femto base stations are installed. This restricts our ability to insert femto base stations, but the achieved penetrations are high enough to observe the benefits of this approach.

\subsubsection{Coverage Area}

The area covered by each sector of a base station is defined as an area where the expected received signal level from the observed sector is the strongest out of all expected signal levels, and greater than $-90 \mathrm{dBm}$. The calculation of the expected received signal level is explained in Section 2.1.

The resulting sectorization is depicted in Figure 3 where only class A base stations are deployed in the region. The white parts are the areas where the received signal level from all base stations is below $-90 \mathrm{dBm}$. There are two reasons for their existence: (1) the observed region contains remote terrains (lakes, forests, mountains) without signal coverage, and (2) at the borders of the observed region we do not have complete information about the base stations outside of the region, which would cover some of the signal-less areas.

Figure 4 shows the case where both class A and class B base stations are deployed in the region. Figure 5 depicts the area covered by 300 femto cells deployed in a network with only class A base stations. 


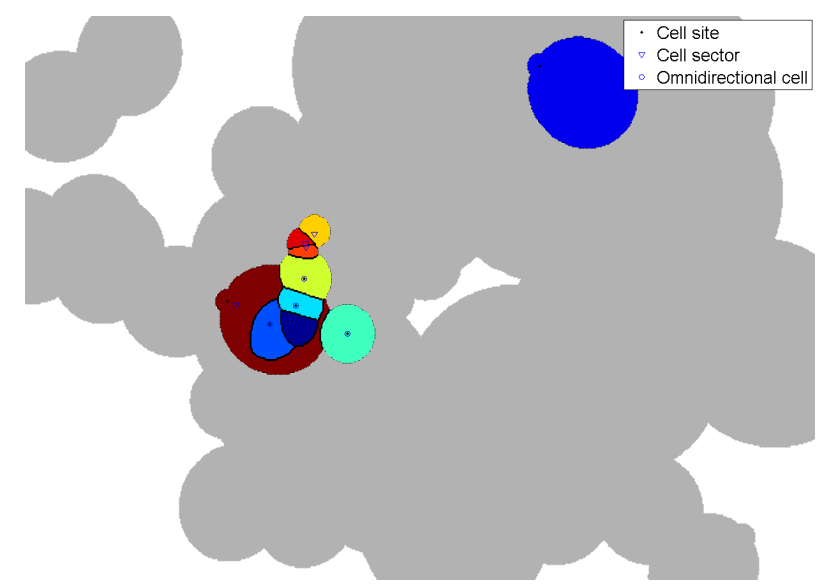

Figure 4: Coverage area of class B (redundant) macro and micro base-station sectors. For comparison, coverage area of class A macro and micro base-station sectors from Figure 3 is depicted in gray.

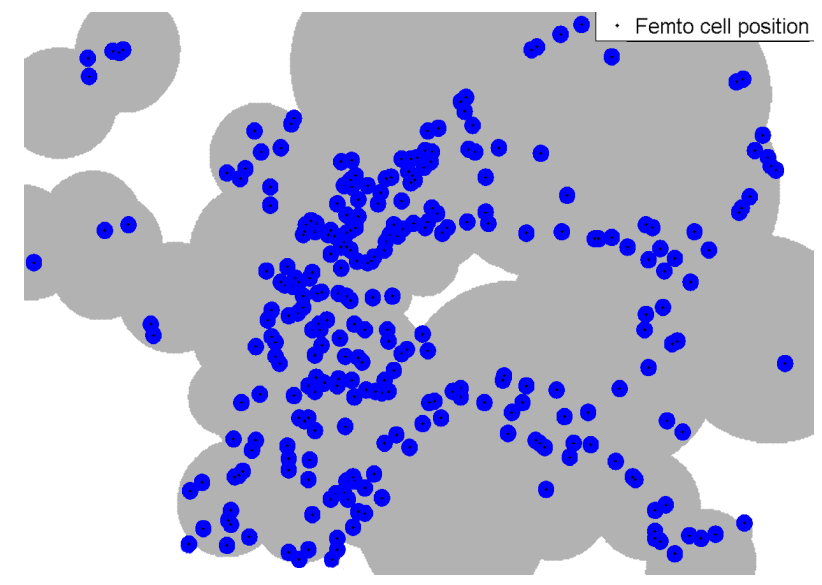

Figure 5: Coverage area of femto base stations. For comparison, coverage area of class A base-station sectors from Figure 3 is depicted in gray.

\subsection{Quality of Service Metric and User Asso- ciation Policy}

A base station serves a user only if it can guarantee a minimum level of quality of service. We consider the expected service time as the metric of quality of service, where a base station accepts a user only if it can serve the user in a bounded time. In particular, we consider that a base-station sector $i$ accepts a service request from a user if its traffic load ( $\rho_{i}$ given by equation (2)) would not grow greater than 0.8 . By applying this constraint, we guarantee that the user will not experience a large service delay as sector $i$ is stable $(i$ is stable for any $\left.\rho_{i} \leq 1\right)$. Note that the service cannot be denied for a user because of lack of the coverage. This is because our model assumes the users are always positioned in one of the class A micro or macro base stations, as defined in Section 2.3.1, and because of our traffic model.

The user association policy is the following: when a user arrives, he selects among all active base stations in the area the one that provides him the largest signal-to-interferenceplus-noise ratio. If this is a femto base station that cannot serve the user without violating the quality of service constraint, he will be forwarded to the best available macro or micro base station. If the user terminal tries to connect to a busy macro or micro base station, he will be blocked.

\section{DEVICE ENERGY PROFILE}

The power consumption of the current technology hardware under maximum load, in watts, is [6]:

$$
P_{m a}^{m a x \_l o a d}=a_{m a} \cdot P_{T X}+b_{m a}
$$

for urban and suburban macro base stations, and

$$
P_{m i}^{\text {max_load }}=a_{m i} \cdot P_{T X}+b_{m i}
$$

for urban micro base stations. The coefficients $a_{m a / m i}$ account for the power consumption that scales with the transmitted power, whereas $b_{m a / m i}$ are power offsets consumed independently of the transmitted power. $P_{T X}$ is the actual transmit power of the base stations and can be calculated in $\mathrm{dBm}$ as follows:

$$
P_{T X}=P_{\text {TXsubcarrier }}-G_{T X}+10 \log _{10} N,
$$

$P_{\text {TXsubcarrier }}$ is defined in Section 2.1, $G_{T X}$ is the antenna gain (15 dB for macro base stations, $2 \mathrm{~dB}$ for micro base stations [6]). $N=300$ is the number of used sub-carriers. For macro base stations, we assume two antennas per sector, hence, the energy consumption of the three-sector macro base stations is calculated using $a_{m a}=22.6$ and $b_{m a}=$ $412.4 \mathrm{~W}[6]$. For a single sector these values are scaled by $1 / 3$. Micro base stations are omnidirectional, hence, we set $a_{m i}=5.5$ and $b_{m i}=32 \mathrm{~W}$, similarly to [6].

The power consumption of a femto base station under maximum load is $15 \mathrm{~W}$ [4], for mobile terminals it is $3 \mathrm{~W}$ under maximum load [7]. We assume that $2 / 3$ of the power consumption of a femto (resp. mobile terminal) is power dependent and $1 / 3$ is power offset consumed independently of the transmit power of the femto (resp. mobile).

The values given are appropriate for the current hardware technology under maximum load [6]. However, more energy efficient devices are expected to appear, so we extend our simulation to more appropriate models for the future.

Assume that time is divided into intervals of one unit and let $\rho$ be the traffic load of a macro/micro base station at a given time slot. $\rho$ (resp. $(1-\rho))$ is the fraction of the time slot that the base station is in active mode (resp. in standby mode). The power consumption of the base station during an active period is calculated in (4) and (5). During the standby period, the power consumption of the base station is equal to the sum of two offsets: one offset that considers inefficiency of output RF amplifiers at zero load [4] and another offset that considers cooling systems and other sources of losses in the base station (e.g. $b_{m a / m i}$ in (4) and (5)). Hence, the power consumption of a base station with a traffic load $\rho$ is:

$$
P_{m a}(\rho)=a_{m a} \cdot P_{T X}[\rho+\alpha(1-\rho)]+\beta b_{m a}
$$

if it is an urban or suburban macro base station and

$$
P_{m i}(\rho)=a_{m i} \cdot P_{T X}[\rho+\alpha(1-\rho)]+\beta b_{m i}
$$

if it is a urban micro base station, all in watts. In above equations, $\alpha(1-\rho) a_{m a} P_{T X}$ and $\alpha(1-\rho) a_{m i} P_{T X}$ represent offsets due to inefficiency of output RF amplifiers of macro and micro base stations during standby period, respectively. 
$\alpha, \beta \in[0,1]$ are coefficients used to model different hardware generations by weighting offsets. In particular, if base stations are strictly energy proportional then $\alpha=\beta=0$ and if their power consumptions are independent from their traffic loads then $\alpha=\beta=1$. In practice, we will be somewhere between these two extreme cases.

For femto base stations, we have

$$
P_{f}(\rho)=10[\rho+\alpha(1-\rho)]+5 \beta,
$$

in watts. For mobile terminals, we have $P_{m}(\rho)=2 \rho+\beta$, in watts (as we consider downlink traffic, mobile terminals are all in a receiving mode and thus the offset due to the inefficiency of RF output amplifiers is set to zero). Note that our main goal in this paper is to compare the energy consumption of femto and macro-micro deployment strategies at the operator side (refer to Section 4); hence, our main results and conclusions do not depend on the energy consumption models we use for mobile terminals and femto base stations.

We introduce four device energy profile models based on four different values of $\alpha$ and $\beta$ to consider all possible hardware generations. For the sake of simplicity, we set $\alpha=\beta$.

- energy independent: $\alpha=\beta=1$. This model represents load independent power consumption devices.

- technology 1: $\alpha=\beta=2 / 3$. This model represents slight energy proportional devices.

- technology 2: $\alpha=\beta=1 / 3$. This model represents high energy proportional devices.

- energy proportional: $\alpha=\beta=0$. This model represents strict energy proportional devices where the offsets are zero.

\section{CAPACITY ADDING STRATEGIES AND PERFORMANCE METRICS}

Consider the network depicted in Figure 3, where only class A base stations are deployed in the area. We compare two strategies that increase the capacity of this network:

- Macro-micro deployment: The capacity of the network is increased by the deployment of redundant class $\mathrm{B}$ macro and micro base stations by the telecom operator. The locations where class B base stations are deployed are shown in Figure 4.

- Femto deployment: The capacity of the network is increased by the deployment of femto base stations by home users as discussed in Section 2.3. Figure 5 depicts the area covered by 300 femto base stations.

We study the energy consumption of these two capacity adding strategies as detailed below. We consider that when a base station (macro, micro, or femto) is deployed in the network, it is always on; hence, we do not apply any on/off scheduling. Note that the nature of class B and femto base stations is similar - they are both redundant and are deployed with the purpose of providing capacity, not coverage.

Let $\beta_{v}=\lambda_{v} \sigma_{v}$ (resp. $\left.\beta_{w}=\lambda_{w} \sigma_{w}\right)$ in bits per second denote the traffic intensity of video (resp. web) users. Average traffic intensity $\beta$ is used to express the average amount of traffic arriving to the network. We define it by formula

$$
\beta=\frac{N_{w} \beta_{w}+N_{v} \beta_{v}}{N_{w}+N_{v}}
$$

where $N_{w}$, resp. $N_{v}$, is the number of arrived web, resp. video, sessions during the simulation.

Several performance metrics are used to compare addmicro and add-femto methods:

- Total energy consumption, $E_{T}$, incorporates the energy consumption of all types of deployed base stations and mobile terminals in the region. It is the sum of the energy spent on operating each device (i.e. macro, micro, and femto base stations and mobile terminals).

- Operator energy consumption, $E_{O}$ : All energy spent on operations, i.e. on macro and micro base stations (of class A or B) deployed in the region.

- Fraction of blocked sessions, $B$, represents sessions blocked on arrival, due to the network reaching the capacity limit.

- Operator-energy-consumption/total-energy-consumption per unit of capacity, $E_{C O} / E_{C T}$ : For a given scheme, we define the network capacity as the highest average traffic intensity $\beta$ for which the fraction of blocked sessions is below $1 \%$, i.e.

$$
\beta_{\max }=\underset{\beta}{\arg \max }\{B(\beta)<1 \%\},
$$

where $B(\beta)$ is the fraction of blocked sessions obtained by injecting traffic with average intensity $\beta$ to the network. The total energy consumption per unit of capacity of the scheme is then defined as

$$
E_{C O}=\frac{E_{O}}{\beta_{\max }},
$$

Similarly, we define $E_{C T}=\frac{E_{T}}{\beta_{\max }}$ as the total energy consumption per unit of capacity.

The reasons we define $E_{C O}$ and $E_{C T}$ are as follows. The network operators consider the blocking probability as a constraint: because a network that blocks large fractions of sessions is not acceptable to them for customer satisfaction and legal reasons. Consequently, $E_{C O}$ and $E_{C T}$ metrics are of great relevance to them. Also, these metrics are very useful to compare different approaches, because they allow us to take into account both energy and QoS, in our case in the form of the fraction of blocked users.

\section{SIMULATION RESULTS}

We first describe our simulation setup. We then discuss the results. The simulation is done in matlab and includes all modeling details explained in Sections 2 and 3.

\subsection{Simulation Setup}

We simulate a network of 76 class A macro and micro basestation sectors, covering a medium-sized city including its outskirts, as depicted in Figure 3. Unless otherwise stated, for simulations including class B base stations, we consider the 10 base-station sectors deployment depicted in Figure 4.

We simulate a time period of one day, beginning at $2 \mathrm{pm}$. During the simulation about 155 thousands of sessions arrive at the network. As described in Section 2.2, based on the trace file we obtained from the network operator, about one third of them are considered as video traffic and the rest of them as web traffic. We experimented with different ratios between video and web traffic and observed similar trends 


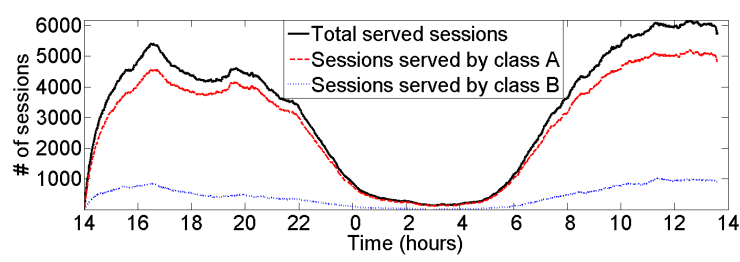

(a) Network of class A and B base stations - total number of sessions served in the network, number of sessions served by non-redundant class A base-station sectors, and number of sessions served by redundant class B base-station sectors.

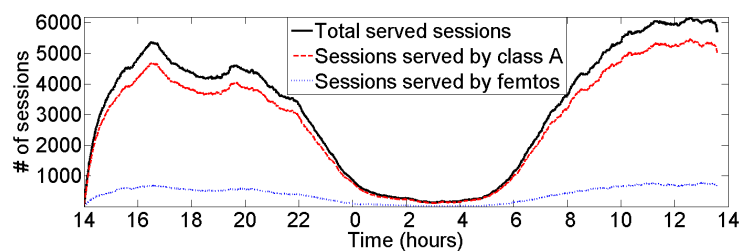

(b) Network of class A and 300 femto base stations - total number of sessions served in the network, number of sessions served by non-redundant class A base-station sectors, and number of sessions served by 300 femto base stations.

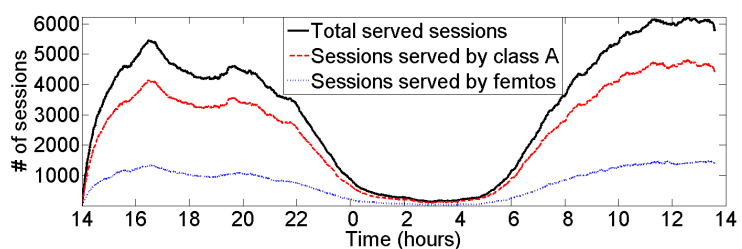

(c) Network of class A and 600 femto base stations - total number of sessions served in the network, number of sessions served by non-redundant class A base-station sectors, and number of sessions served by 600 femto base stations.

Figure 6: Representative course of simulation for three scenarios - (a) class A plus class B, (b) class A plus 300 femto base stations, (c) and class A plus 600 femto base stations.

in the results, for brevity we present results for one traffic mix only.

We examine results for different traffic intensities $\beta$. For video sessions we use $\beta_{v}=25000,50000, \ldots, 200000 \mathrm{bps}$, for web sessions we assume one tenth of it.

\subsection{Single Simulation}

For each simulation, the simulation time is split into time slots, during which the situation in the network does not change, i.e. the splitting is done on each session arrival or departure. On session arrival, the user association policy described in Section 2.4 is applied. For each of the time slots, a number of values is computed - the current rate of each ongoing session, the current traffic load of each base-station sector, the current power consumption of each base station, mobile terminal, etc. A representative course of a simulation is visualized in Figure 6 for macro-micro and femto deployment approaches for $\beta=100000 \mathrm{bps}$. For the illustration, three cases are represented: (1) a network with class A and class B base stations (subfigure 6(a)), (2) a network with class A and 300 femto base stations (subfigure 6(b)) and (3) a network with class $\mathrm{A}$ and 600 femto base stations (sub-

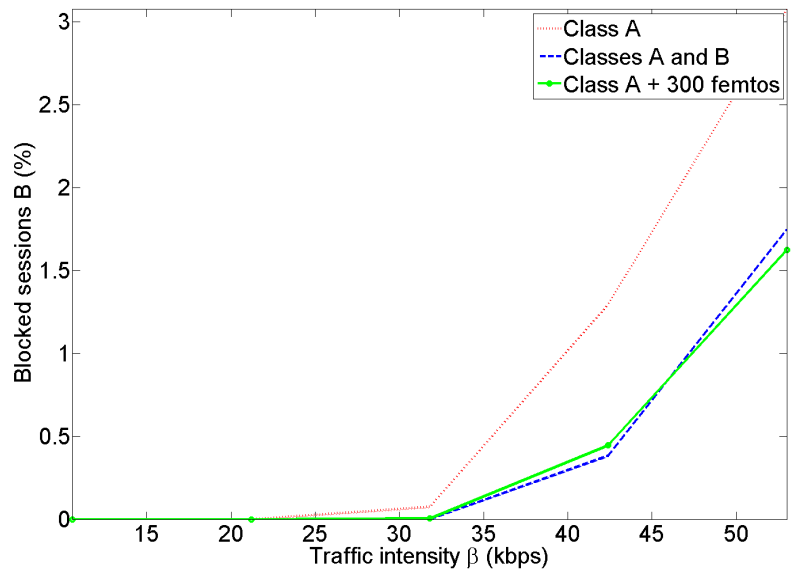

Figure 7: Blocking probability versus traffic load for three described scenarios (class A base stations only, macro-micro deployment and femto deployment). We see that the blocking probability $B(\beta)=1 \%$ is achieved for traffic intensities $\beta=39.9 \mathrm{kbps}$ for class A base stations only and $\beta=47.5 \mathrm{kbps}$ for two other cases.

figure $6(\mathrm{c})$ ). We observe that class B base stations serve about $15 \%$ of sessions; $13 \%$ of sessions are served by femto base stations when 300 of them are deployed in the network; and the number of served sessions is almost doubled when 600 femto base stations are deployed in the network. However, our simulation results show that this linear relationship between the number of deployed femto base stations and the number of served sessions by femto base stations holds for only low penetrations of femto deployments, whereas for high penetrations this relationship is sub-linear. This is in accordance with results in [5] and differs from [4] as it assumes a linear relationship between the number of deployed femto base stations and the number of served sessions by femto base stations independent of the penetration.

\subsection{Energy Consumption Comparision of Macro- Micro and Femto Deployment Strategies}

Our goal is to compare the operator and total energy consumption of macro-micro and femto deployment strategies. In particular, we simulate the following scenarios

- a network of only class A base stations,

- a network of class A and B base stations,

- a network of class A and femto base stations.

For the last case, we consider different numbers of femto base stations. However, in this section we show the results only for 300 femto base stations, as they yield similar capacity gain as deployment of class $B$ base stations.

Figure 7 depicts the blocking probability versus traffic load for the above described scenarios. Note that the blocking probability is the same for all energy profile models. Figure 8 depicts the operator power consumption versus a traffic load for three scenarios described above and for four energy profile models proposed in Section 3. We observe:

- The capacity of the network where only class A base stations are deployed is $\beta=39.9 \mathrm{kbps}$. 


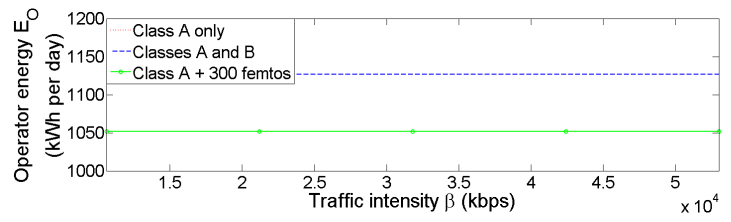

(a) Energy independent energy profile

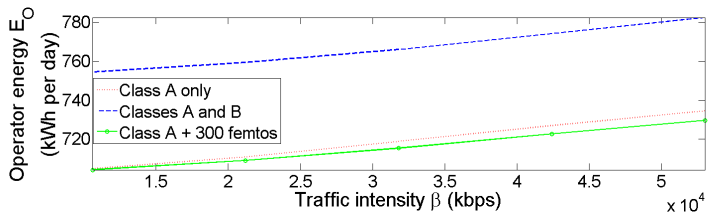

(b) Technology 1 energy profile

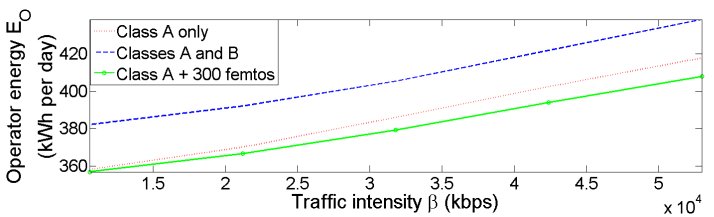

(c) Technology 2 energy profile

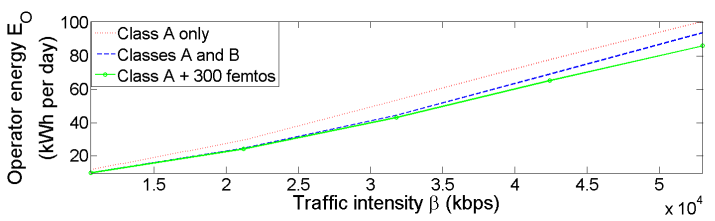

(d) Energy proportional energy profile

Figure 8: Operator energy consumption for three described scenarios (class A base stations only, macro-micro deployment and femto deployment) and four energy models. Note that the area of interest is when $B(\beta)<1 \%$, i.e. for traffic intensities up to $\beta=39.9 \mathrm{kbps}$ for class A base stations only and up to $\beta=47.5 \mathrm{kbps}$ for two other cases.

- The capacity of the network is increased by $19 \%$ by deployment of class B base stations. A similar capacity gain can be achieved by deployment of 300 femto base stations in the network.

- There is no significant difference between operator energy consumption of femto and macro-micro deployment strategies. Even in the worst case (subfigure $8(\mathrm{a})$ ), the difference is less than $7 \%$. This differs from the previous findings in [4], which compares these capacity adding strategies, and finds that femto deployment is considerably more energy efficient. Note that to calculate $E_{O}$, we ignore the energy consumption of femto base stations where femto deployment strategy is applied. However, the power consumption of class $\mathrm{B}$ base stations is included in $E_{O}$ when macro-micro deployment strategy is used.

Figure 9 compares the operator-energy-consumption per unit of capacity for macro-micro and femto deployment strategies. We observe that, for all energy models, using femto base stations is around $7 \%$ more energy efficient compared to the macro-micro deployment. We consider these savings as negligible, keeping in mind that operators would prefer to have a smaller number of class B base stations under their direct control rather than a large number of unreliable femto

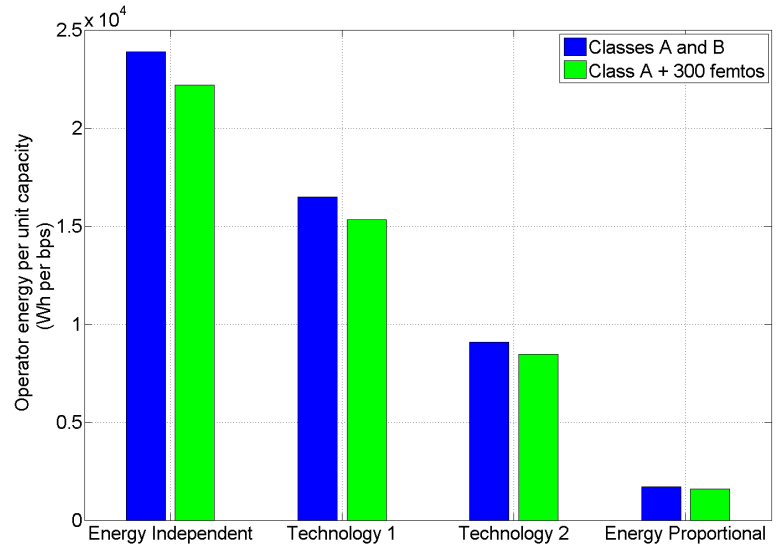

Figure 9: $E_{C O}$ (operator-energy-consumption per unit of capacity) for two described scenarios (macro-micro deployment and femto deployment) and for four different energy models.

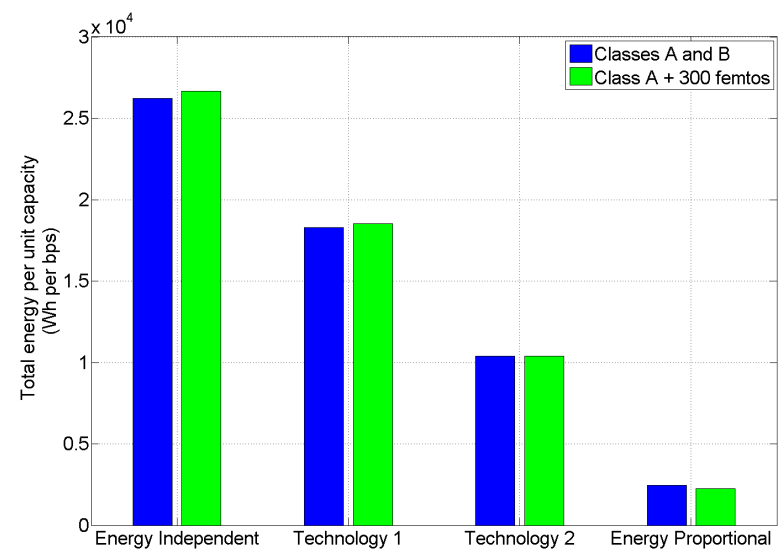

Figure 10: $E_{C T}$ (total-energy-consumption per unit of capacity) for two described scenarios (macro-micro deployment and femto deployment) and for four different energy models.

base stations (users could turn them off anytime). Moreover, as stated above, to calculate $E_{O}$ we ignore energy consumption of femto base stations. Figure 10 compares the total-energy-consumption per unit of capacity for macromicro and femto deployment strategies. Here we observe that, from the point of view of society, macro-micro deployment is even more energy efficient in some cases. Femto deployment is advantageous only in very energy proportional energy models that are unrealistic, but even in this case the gain is negligible.

Moreover, from the results represented in Figures 9 and 10 we observe that the significant energy saving can be achieved by using more energy proportional devices. Hence, it may be more beneficial for the operator to invest in the equipment that is more energy proportional (change the hardware) rather than to change the way networks are managed, which would be imposed by introducing femto base stations.

\subsection{Effect on Mobile Terminals}

Due to the battery constraints of mobile terminals, it is 


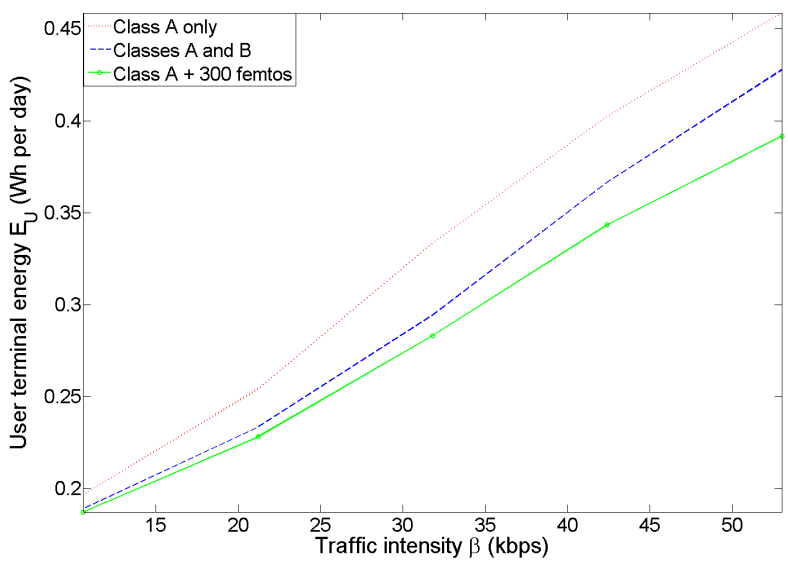

Figure 11: Average-energy-consumption per a served session at mobile devices, for femto and macro-micro deployment approaches, for Technology 2 energy model. Note that the area of interest is when $B(\beta)<1 \%$, i.e. for traffic intensities up to $\beta=39.9 \mathrm{kbps}$ for class A base stations only and up to $\beta=47.5 \mathrm{kbps}$ for two other cases.

very important not to increase the users' energy consumption. Figure 11 presents the average-energy-consumption per a served session (not blocked) for Technology 2 energy profile (other profile models show similar trend). We observe that femto deployment has a positive effect on mobile terminal batteries, in accordance with findings in [9]. This is due to the better SINR, and consequently higher rate, obtained when using a local femto base station instead of a distant macro or micro base station. Macro-micro deployment also has positive effects on mobile-terminal energy consumption. There is no significant difference between energy consumption of mobile terminals when macro-micro and femto deployment strategies are applied.

\subsection{Effect of Femto Base-Station Penetration}

There is a strong correlation between the number of femto base stations deployed and the energy saving they bring to the network. In this section, we consider different numbers of femto base stations $N_{f}=0,200, \ldots, 1000$. Figure 12 depicts the operator and total-energy-consumption per unit of capacity, i.e. $E_{C O}$ and $E_{C T}$, for femto deployment strategy versus $N_{f}$ for four energy profile models. We observe that $E_{C O}$ and $E_{C T}$ are both decreasing in $N_{f}$.

We see from Figure 12 that for higher penetrations the positive effects of femto base stations on $E_{C T}$ diminishes. Furthermore, we expect that it would be even outweighed by the power required to keep femto base stations active. Moreover, with higher penetrations of femto base stations, interference is becoming more serious. However, we could not verify these hypotheses as our two-dimensional interference model prevented us from adding more femto base stations without sacrificing faithfulness, as explained in Section 2.3. This is because in this two-dimensional setting we can not distinguish between two femto base stations in the same building on different floors.

Applying on/off strategies may decrease the energy consumption of femto base stations and hence decrease the total energy consumption per unit of capacity when femto base stations are deployed. $E_{C T}$ and $E_{C O}$ curves in Figure 12 provides upper bound and lower bounds for total-energyconsumption per unit of capacity of such an on/off strategy. Note that on/off strategies can also be applied to class B base stations as they do not contribute to the coverage of class A base stations and hence can be turned off without losing the coverage in the region.

In Figure 12, we also show $E_{C O}$ and $E_{C T}$ for macro-micro deployment strategy. Similarly to what we concluded in Section 5.3, macro-micro deployment strategy and deployment of 300 femto base stations provide similar capacity enhancement and comparable energy consumption. Note that the nature of class B and femto base stations is similar, keeping in mind that they are both redundant and are deployed with the purpose of providing capacity and not coverage. Hence, we strongly believe that higher numbers of class B base stations and higher penetrations of femto base stations will bring similar benefits. However, network planning is required to decide about positions of additional class B base stations, which is out of scope of this paper. We relegate this to future work.

Also, similar to our previous conclusion in the Subsection 5.3, we can observe from Figure 12 that the energy saving that could be obtained by making macro and micro base stations more energy proportional is much higher than the energy saving of femto deployment.

\section{SUMMARY AND DISCUSSION}

We have quantified the energy consumptions of two alternative strategies to increase capacity in future LTE networks: (1) deployment of redundant micro base stations by telecom operators at locations where traffic load is high and (2) deployment of femto base stations by home users. We focus on downlink traffic. We have illustrated that these two strategies have similar energy consumption, which differs from the previous findings in [4], where it states that deployment of femto base stations was considerably more energy efficient.

In this paper, we have not considered any adaptive power control mechanisms $[18,19]$ or fractional frequency reuse schemes [20,21], and we assumed that there was no cooperation among base stations [10,22]. By applying any of these mechanisms, we can increase the capacity of the network and make it more energy efficient. However, it is much easier to apply these mechanisms in a network consisting of a few macro and micro base stations than in a network of hundreds of femto base stations, especially for centralized schemes such as COMP [22].

Based our results, we believe that it is more reasonable for a telecom operator to apply the macro-micro deployment strategy because (1) it has the same energy consumption as femto deployment method; (2) it is much easier to control and manage a few macro and micro base stations than hundreds of femto base stations; and (3) physical layer capacity enhancing schemes, such as those proposed in [19-22], can be implemented easier in a network with only micro and macro base stations. Moreover, we have shown that the energy saving that could be obtained by making macro and micro base stations more energy proportional is much higher than the energy saving of femto deployment. Hence, it may be more beneficial for the operator to invest in the more energy proportional equipments (i.e. change the hardware), rather than to change the way networks are managed, which would be imposed by introducing femto base stations. 


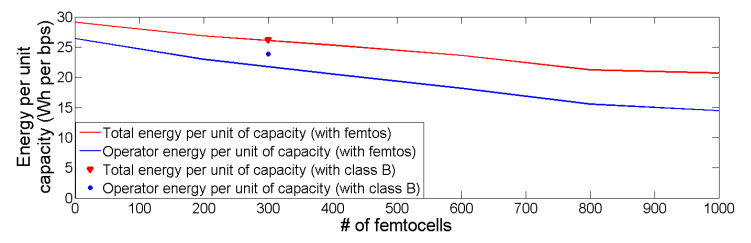

(a) Energy independent energy profile

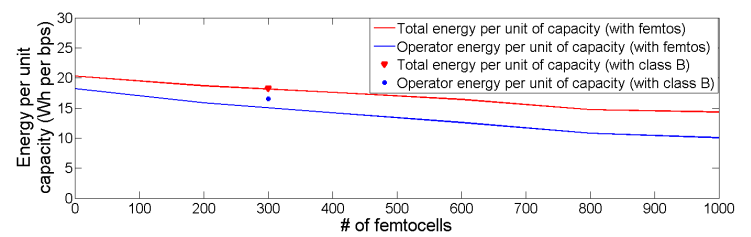

(b) Technology 1 energy profile

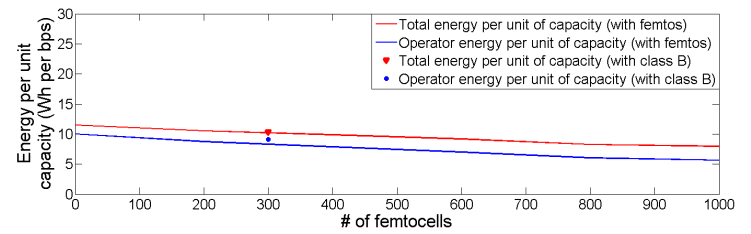

(c) Technology 2 energy profile

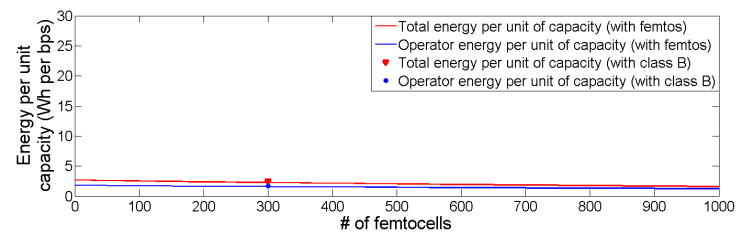

(d) Energy proportional energy profile

Figure 12: $E_{C O}$ and $E_{C T}$ (operator and total-energyconsumption per unit of capacity) for femto deployment strategy versus $N_{f}$ for four different energy models. We also depict $E_{C O}$ and $E_{C T}$ for macro-micro deployment strategy.

Our study has some limitations that we intend to focus on in future work. Given a 2D surface representation, the number of deployed femto base stations is artificially constrained - a 3D representation would allow for more femto base stations to be deployed. To obtain the complete picture, the uplink traffic should be added into consideration. Finally, statically modeled users need to be replaced with realistic mobility models [23] and the resulting effect of inter-cell handovers must be considered. We have not considered joint deployment of class B and femto base stations in this paper - we were interested mainly in comparing the two cases.

\section{REFERENCES}

[1] H.-O. Scheck. ICT \& wireless networks and their impact on global warming. In European Wireless Conference, 2010.

[2] Cisco. Cisco visual networking index: Global mobile data traffic forecast update, 2009-2014. White paper, 2010.

[3] S. R. Saunders, S. Carlaw, A. Giustina, R. R. Bhat, V. S. Rao, and R. Siegberg. Femtocells: Opportunities and Challenges for Business and Technology. Wiley, 2009.
[4] H. Claussen, L. T. W. Ho, and F. Pivit. Leveraging advances in mobile broadband technology to improve environmental sustainability. Telecommunications Journal of Australia, 59(1), 2009.

[5] Benyuan Liu and Don Towsley. A study of the coverage of large-scale sensor networks. In In Proceeding of MASSO4.

[6] A.J. Fehske, F. Richter, and G.P. Fettweis. Energy efficiency improvements through micro sites in cellular mobile radio networks. In GLOBECOM Workshops, 2009.

[7] P. Somavat, S. Jadhav, and V. Namboodiri. Accounting for the energy consumption of personal computing including portable devices. In Proceedings of e-Energy 2010, April 2010.

[8] Ericsson. Sustainable energy use in mobile communications. White paper, 2007.

[9] Femto Forum. http://www . femtoforum.org.

[10] 3GPP Technical Specification Group Radio Access Network. Further advancements for E-UTRA physical layer aspects (Release 9). Technical report, 2010.

[11] OFCOM. Radio transmitters locations in Switzerland http://www . funksender.ch/webgis/bakom.php, July 2010.

[12] HNB and HNB-Macro Propagation Models. Technical report, Qualcomm Europe, 2007.

[13] 3GPP Technical Specification Group Radio Access Network. Spatial channel model for multiple input multiple output (MIMO) simulations (Release 9). Technical report, 2009.

[14] T. Bonald and N. Hegde. Capacity gains of some frequency reuse schemes in ofdma networks. In GLOBECOM, 2009.

[15] Jean-Yves Le Boudec. Performance evaluation of computer and communication systems. EPFL Press, 2010 .

[16] Thomas Bonald. Insensitive queueing models for communication networks. In VALUETOOLS, 2006.

[17] T. Bonald and A. Proutière. On performance bounds for the integration of elastic and adaptive streaming flows. SIGMETRICS Perform. Eval. Rev., 32, 2004.

[18] M. Chiang, P Hande, T Lan, and CW Tan. Power control in wireless cellular networks. now Publishers Inc, 2008.

[19] G. Miao, N. Himayat, G.Y. Li, A.T. Koc, and S. Talwar. Interference-aware energy-efficient power optimization. In $I C C, 2009$.

[20] Y. M. Kwon, O. K. Lee, J. Y. Lee, and M. Y. Chung. Power control for soft fractional frequency reuse in ofdma system. In ICCSA, 2010.

[21] C. Y. Wong, R. S. Cheng, K. B. Letaief, and R. D. Murch. Multiuser ofdm with adaptive subcarrier, bit, and power allocation. IEEE JSAC, 17, 1999.

[22] G. J. Fochini, K. Karakayali, and R. A. Valenzuela. Coordinating multiple antenna cellular networks to achieve enormous spectral efficiency. IEEE Proc.-Commun., 153(4), 2009.

[23] Jean-Yves Le Boudec and Milan Vojnovic. The random trip model: Stability, stationary regime, and perfect simulation. IEEE/ACM Transactions on Networking, 14, 2006. 